\title{
The effect of caffeine on the ventilatory response to hypercarbia in preterm infants
}

\author{
Thomas Rossor ${ }^{1}$, Ravindra Bhat ${ }^{2}$, Kamal Ali ${ }^{2}$, Janet Peacock ${ }^{3}$, Gerrard F Rafferty ${ }^{1}$ and Anne Greenough ${ }^{1}$
}

BACKGROUND: We tested the hypotheses that caffeine therapy would increase the ventilatory response to hypercarbia in infants above the effect of maturation and those with a weaker ventilatory response to hypercarbia would be more likely to subsequently develop apnea that required treatment. METHODS: Infants born at less than 34 weeks of gestation underwent a steady-state hypercarbic challenge using 0,2 , and $4 \%$ carbon dioxide soon after birth that was repeated at weekly intervals. The results of the initial study were compared between infants who did or did not subsequently develop apnea requiring treatment with caffeine.

RESULTS: Twenty-six infants born at a median gestation of 32 (range 31-33) weeks were assessed. Caffeine administration was associated with an increase in $\mathrm{CO}_{2}$ sensitivity, and the mean increase was $15.3(95 \% \mathrm{Cl}: 1-30) \mathrm{ml} / \mathrm{kg} / \mathrm{min} / \% \mathrm{CO}_{2}$. Fourteen infants subsequently developed apnea treated with caffeine. After controlling for gestational age and birth weight, they had significantly lower carbon dioxide sensitivity at their initial study compared with those who did not require treatment.

CONCLUSION: Caffeine administration was associated with an increase in the ventilatory response to hypercarbia. An initial weaker ventilatory response to hypercarbia was associated with the subsequent development of apnea requiring treatment with caffeine.

A pnea of prematurity is a common problem in prematurely born infants (1) occurring most often in those born very prematurely (2). It is possible that a reduced chemoresponse to hypercarbia may contribute to the development of apnea (1). Indeed, at approximately 1 week of age, 18 infants with significant apnea (a mean of 32 apneas lasting longer than $20 \mathrm{~s}$ per day) had a reduced ventilatory response to carbon dioxide $\left(\mathrm{CO}_{2}\right)$ compared with 18 matched controls without apnea (3).

Caffeine, a methylxanthine, reduces the frequency of apneas in preterm infants (4). It increases the ventilatory response to hypercarbia in animals (5) and adults (6,7). In anesthetized cats, caffeine administration resulted in an increase in minute ventilation, as well as an increased ventilatory response to inspired $\mathrm{CO}_{2}$ (5). Administration of theophylline, another methylxanthine, to ten infants with a median gestational age of 30 weeks and apnea was associated with an increase in minute volume in response to breathing $3 \% \mathrm{CO}_{2}$ and lower end-tidal $\mathrm{CO}_{2}$ levels (8). The measurements, however, were made before and between 2 and 4 days after administration of theophylline and no attempt was made to determine whether the effects were due to maturation rather than theophylline administration per se (8). No study has investigated whether caffeine administration influences the ventilatory response to hypercarbia of prematurely born infants. We hypothesized that the ventilatory response to hypercarbia would be increased after administration of caffeine, and any effect would be additional to the effect of increasing maturation.

Furthermore, we postulated that those infants who went on to require treatment with caffeine compared with those who did not, when first studied, have a lower response to a $\mathrm{CO}_{2}$ challenge. We have undertaken a longitudinal study to test these hypotheses.

\section{METHODS}

The study was performed at King's College Hospital NHS Foundation Trust between May 2013 and August 2015. Infants were eligible for entry into the study if they were born at less than 34 weeks of gestation, did not require respiratory support, and were less than $72 \mathrm{~h}$ of age. Infants only entered into the study if they had not received caffeine. Infants with major congenital abnormalities were excluded from the study. A hypercapnic challenge was undertaken and repeated at weekly intervals, until the infants were discharged from the neonatal unit. Infants who developed clinically significant apnea (see later) during the course of the study were treated with caffeine. Parent(s) gave informed, written consent for their infant to take part in the study, which was approved by the London Bromley Research Ethics Committee.

\section{The Hypercapnic Challenge}

The test gas was delivered using an open-circuit system via a nasal mask and pneumotachograph. The pneumotachograph (Mercury F10L, G M Instruments, Kilwinning, Scotland) that had a dead space of $0.8 \mathrm{ml}$ and resistance of $0.86 \mathrm{~mm}$ of $\mathrm{H}_{2} \mathrm{O} / \mathrm{l} / \mathrm{min}$, was connected to a soft latex nasal mask (Neomask, Draeger, Germany) using a snugly fitting connector. The pneumotachograph was connected to a differential pressure transducer-amplifier system (Gould model 134615-70, Cleveland, $\mathrm{OH})$, generating an analog signal proportional to

\footnotetext{
'Department of Women and Children's Health, School of Life Course Sciences, Faculty of Life Sciences and Medicine, King's College London, London, UK; ${ }^{2}$ Neonatal Intensive Care Centre, King's College Hospital NHS Foundation Trust, London, UK; ${ }^{3}$ School of Population Sciences, School of Population Sciences and Health Services Research, King's College London, London, UK. Correspondence: Anne Greenough (anne.greenough@kcl.ac.uk)

Received 21 September 2017; accepted 15 February 2018; advance online publication 23 May 2018. doi:10.1038/pr.2018.48
} 


\section{Caffeine, $\mathrm{CO}_{2}$ sensitivity, and apnea $\mid$ Articles}

airflow. The mask was placed over the infant's nose. A nasal mask (dead space $7.5 \mathrm{ml}$ ) was used to avoid the stimulation that can result if a facemask is used (9). A seal was achieved by gentle pressure and confirmed by demonstration of no leak (there was no discrepancy between the inspired and expired tidal volumes, as displayed in real time on the computer screen). We monitored the flow waveforms to ensure that no airway obstruction was occurring. The distal end of the pneumotachograph was connected to a two-way non-rebreathing valve that separated inspired from expired gas, such that the controlled mixture of gases was inspired by the infant and expiration was to the outside air. A constant flow of medical air was delivered to the inspiratory port of the valve via a wide-bore $(20 \mathrm{~mm})$, lowresistance tubing to eliminate any dead space. The inspired air could be enriched with a variable concentration of $\mathrm{CO}_{2}$ from a cylinder. Gas was continuously sampled from the nasal mask through a finebore catheter at the rate of $180 \mathrm{ml} / \mathrm{min}$ using a capnograph ( $\mathrm{CO}_{2} \mathrm{SMO}$ capnograph) (Respironics UK, Chichester, UK). The $\mathrm{CO}_{2}$ content of the sampled gas was determined by infrared spectroscopy and an analog signal proportional to the $\mathrm{CO}_{2}$ concentration generated. Oxygen saturation was measured using a pulse oximeter (Masimo rainbow SET Pulse Oximetry) attached to the foot of the infant.

Following a 5-min period of breathing medical air, the hypercarbic challenge was delivered through the equipment described above. $\mathrm{CO}_{2}$ was delivered from a cylinder of $\mathrm{CO}_{2} 20 \%$ balanced with medical air (BOC), via a low-flow meter. Minute volume was measured during exposure to three levels of $\mathrm{CO}_{2}$ ( $0 \%$ (baseline), $2 \%$, and $4 \%$ ). These levels were chosen as they have been previously demonstrated to result in changes in respiration without significant behavioral arousal (10-13). Each mixture of $\mathrm{CO}_{2}$ was titrated using a low-flow meter attached to the $\mathrm{CO}_{2}$ regulator. The capnograph read out was used to determine when a stable inspiratory $\mathrm{CO}_{2}$ concentration within the delivery tubing had been achieved.

The fraction of inspired oxygen was not monitored or controlled; however, addition of a maximum of $4 \% \mathrm{CO}_{2}$ would result in a potential reduction in the inspired oxygen of less than $1 \%$.

The infant breathed the air/ $\mathrm{CO}_{2}$ mixture for at least $5 \mathrm{~min}$ to allow ventilation and $\mathrm{ETCO}_{2}$ to reach a stable state, as assessed from the real-time display using the Spectra software (Grove Medical, London, $\mathrm{UK})$. That duration is in keeping with the time used in previous studies (10). Neither transcutaneous $\mathrm{CO}_{2}$ monitoring nor blood gas analysis was performed. The order of administration of the test gases was randomized between infants. All measurements were made during quiet sleep, as determined by Prechtl's criteria (14). When arousal occurred, the measurement stopped until the infant returned to quiet sleep and then the measurement began again.

Respiratory flow and gas concentration were acquired and displayed in real time on a PC computer running Spectra software (Grove Medical) with $100-\mathrm{Hz}$ analog-to-digital sampling (PCIMIO-16XE-50, National Instruments, Austin, TX). Breath-by-breath data were exported from Spectra software to Microsoft Excel 2011 (Microsoft). Minute volume was calculated from the last minute of exposure at each level of $\mathrm{CO}_{2}$. $\mathrm{CO}_{2}$ sensitivity was calculated as the gradient of a line of best fit through a plot of the minute ventilation at each of the three inspired $\mathrm{CO}_{2}$ levels $\left(0,2\right.$, and $\left.4 \% \mathrm{CO}_{2}\right) \cdot \mathrm{CO}_{2}$ sensitivity was expressed as $\mathrm{ml} / \mathrm{kg} / \mathrm{min} / \% \mathrm{CO}_{2}$, that is, the change in minute volume per $1 \%$ change in the inspired $\mathrm{CO}_{2}$.

\section{Polysomnography}

Polysomnography was performed at the initial study to determine whether the infant was having apneas. A commercially available Alice 4 sleep study unit (Profile Vio-systems, Bognor Regis, UK), the Alice 5 firmware upgrade was used. Abdominal and thoracic movements were assessed using stretch-sensitive piezoelectric respiratory bands. Oral and nasal airflow were measured using the analog output of the pneumotachograph described above. An electrocardiogram was recorded using single-use bipolar electrodes. Two activity meters were attached, one to an arm and the other to a leg to record limb movements. Oxygen saturation was continuously monitored using a pulse oximeter (Masimo rainbow SET Pulse Oximetry). The data were incorporated into the Alice sleep system using an auxiliary input.

The Alice sleep system was connected to a PC that was used to display the recording in real time and store data. The infant was monitored by a video camera throughout the study with recordings stored on the PC. These recordings were used to assess sleep state and evaluate for artifacts, such as awakenings or handling of the infant. Apneas were defined as cessation of respiratory airflow of $5 \mathrm{~s}$ in duration (15). This definition has been used in the preterm population (16) and ensures a sensitive assessment of respiratory pauses that may not be detected by other definitions (17).

They were classified as obstructive if there was no airflow despite chest and abdominal wall movements; central if there was no nasal airflow and an absence of chest and abdominal wall movements; and mixed if there was a combination of central and obstructive apneas. For each classification, apnea-associated changes in heart rate and oxygen saturations were recorded. The apnea index (the frequency of apneas per hour) was calculated.

\section{Caffeine administration}

The decision to treat with caffeine was made by the lead clinician without knowledge of the results of the polysomnography or hypercarbic challenge. The criteria for treatment with caffeine was apnea lasting for more than $20 \mathrm{~s}$ or more than $10 \mathrm{~s}$ if associated with oxygen desaturation to less than $90 \%$ or bradycardia, as demonstrated by routine clinical monitoring (18). A loading dose of $20 \mathrm{mg} / \mathrm{kg}$ caffeine citrate was administered intravenously, followed by maintenance dose of $10 \mathrm{mg} / \mathrm{kg}$ every $24 \mathrm{~h}$ given either intravenously or enterally. Caffeine therapy discontinued at 34 weeks of corrected gestational age, or earlier if the infant had been without apneas for at least 1 week.

\section{Data collection}

Birth weight, gestational age at birth, and maternal age were recorded. Infants were considered to have been exposed to antenatal corticosteroids if at least one dose of corticosteroids was given to their mother $24 \mathrm{~h}$ before delivery. A diagnosis of chorioamnionitis was made if the diagnosis was documented in the obstetric records or if the mother had received parenteral antibiotics because of the clinical features of chorioamnionitis (fever, fetal tachycardia, or offensive liquor). At each study, the current weight, corrected gestational age and postnatal age, and caffeine treatment were documented from the medical records.

\section{Statistical Analysis}

The results were visually assessed for normal distribution using histograms and normality plots and shown to be normally distributed. Hence, differences in outcomes were assessed for statistical significance using Fisher's exact test or the Student's $t$ test as appropriate. Comparison of the results of the longitudinal measurements of infants receiving or not receiving caffeine was performed using a paired $t$-test.

Linear-mixed models with fixed effects were developed to account for repeated measures, with $\mathrm{CO}_{2}$ sensitivity as the dependent variable. Corrected gestational age was entered as a covariate, when determining the effect of corrected gestational age on $\mathrm{CO}_{2}$ sensitivity, with caffeine as a factor to determine the additional effect of caffeine on $\mathrm{CO}_{2}$ sensitivity.

Differences in results of the initial challenge between those who went on to require treatment with caffeine and those who did not were assessed using regression analysis. Adjustment was made for birth weight and gestational age by fitting those variables as covariates. Adjusted means are marginal estimates for the dependent variable derived from the regression model at the mean value of the covariates: birth weight and gestational age.

Analyses were conducted using SPSS 22 (IBM). 


\section{Articles | Rossor et al.}

\section{Sample Size}

A sample size of two groups of 15 would allow detection of a twosided difference between caffeine and nontreated infants of a difference equivalent to one standard deviation to be detected by $90 \%$ power. That difference had been detected in the ventilatory response to added dead space (primarily a hypercarbic challenge) between newborns of smoking and nonsmoking mothers (19).

\section{RESULTS}

Twenty-six infants born at a median gestation of 32 (range 31-33) weeks and a median birth weight of 1,590 (range

Table 1. Demographics of the study population according to the development of significant apnea

\begin{tabular}{lccccc}
\hline & $\begin{array}{c}\text { No significant } \\
\text { apnea }\end{array}$ & & $\begin{array}{c}\text { Significant } \\
\text { apnea }\end{array}$ & P-value \\
\cline { 2 - 2 } \cline { 5 - 5 }$n$ & 12 & & 14 & \\
\hline Birth weight (g) & $1,730(340)$ & & $1,450(290)$ & 0.03 \\
Gestational age (weeks) & $32.7(0.5)$ & & $32.2(0.7)$ & 0.06 \\
Maternal age (years) & $32(7)$ & & $30(6)$ & 0.45 \\
Antenatal steroids & 10 & & 13 & 0.58 \\
Chorioamnionitis & 1 & & 3 & 0.60 \\
Cesarean section & 10 & & 10 & 0.65 \\
Sex (F) & 6 & & 5 & 0.69 \\
Birth weight $<10$ th & 3 & & 6 & 0.43 \\
centile & 10 & & 9 & 0.39 \\
$\begin{array}{l}\text { Singleton } \\
\text { Apgar at } 5 \text { min }\end{array}$ & $10(9-10)$ & $9(8-10)$ & 0.37 \\
$\begin{array}{l}\text { Supplementary oxygen } \\
>12 \mathrm{~h}\end{array}$ & 7 & & 7 & 0.71 \\
$\begin{array}{l}\text { Positive pressure } \\
\text { support }>12 \mathrm{~h}\end{array}$ & 3 & & 5 & 0.68 \\
\hline
\end{tabular}

Data are presented as mean (SD) if normally distributed; median (range); or $n$.
840-2,200) g were recruited into the study. They were first studied at a median 22 (range 6-69) h after birth. Ninety-two studies, each comprising exposure to the three levels of $\mathrm{CO}_{2}$, were performed with a median of four (range 1-6) studies per infant. Fourteen infants developed significant apnea and were treated with caffeine (Table 1). The infants who went on to develop significant apnea had significantly lower birth weight $(P=0.03)$ (Table 1). At the initial study, there was no difference in the apnea index between those infants who went on to develop significant apnea and those who did not (Table 2). $\mathrm{CO}_{2}$ sensitivity was significantly higher following caffeine administration $(n=14 ;$ mean difference $41 \mathrm{ml} / \mathrm{kg} /$ $\left.\min / \% \mathrm{CO}_{2} ; 95 \% \mathrm{CI} 26-57 ; P<0.001\right)$, and the measurements were made 1 week apart (Figure 1). Baseline minute volume while breathing air did not differ significantly between the two measurements (Figure 2).

$\mathrm{CO}_{2}$ sensitivity was significantly lower after discontinuing caffeine therapy ( $n=11$; mean difference $15 \mathrm{ml} / \mathrm{kg} / \mathrm{min} / \%$ $\mathrm{CO}_{2} ; 95 \% \mathrm{CI} 1-29 ; P=0.036$ ) (Figure 3). The measurements were made 1 week apart.

A linear-mixed model of the results from infants who did not receive caffeine $(n=12)$ demonstrated that increased corrected gestational age resulted in an increase in $\mathrm{CO}_{2}$ sensitivity. A 1-week increase in the corrected gestational age correlated with an increase in $\mathrm{CO}_{2}$ sensitivity of $9.5 \mathrm{ml} / \mathrm{kg} /$ $\min / \% \mathrm{CO}_{2}$ (95\% CI: 4.3-14.6; $\left.P<0.001\right)$. When all the infants were included $(n=26)$ in the analysis and caffeine was introduced as a factor, both corrected gestational age and caffeine significantly contributed to a linear-mixed model for $\mathrm{CO}_{2}$ sensitivity. The estimated effect of corrected gestational age was an increase of $10.0 \mathrm{ml} / \mathrm{kg} / \mathrm{min} / \% \mathrm{CO}_{2}$ (95\% CI: $5.4-$ $14.5 ; P<0.001)$ per week. The estimated marginal mean effect

Table 2. Results of the initial study, grouped according to significant apnea status

\begin{tabular}{|c|c|c|c|}
\hline & No significant apnea & Significant apnea & $P$-value \\
\hline$n$ & 12 & 14 & \\
\hline \multicolumn{4}{|c|}{ Baseline minute volume ( $\mathrm{m} / \mathrm{kg} / \mathrm{min})$} \\
\hline Mean $(95 \% \mathrm{Cl})$ adjusted $^{\mathrm{a}}$ & $446(356-537)$ & $413(330-496)$ & 0.61 \\
\hline \multicolumn{4}{|l|}{ Baseline end-tidal $\mathrm{CO}_{2}(\%)$} \\
\hline Mean $(95 \% \mathrm{Cl})$ adjusted $^{\mathrm{a}}$ & $4.1(3.6-4.6)$ & $4.4(4.0-4.9)$ & 0.36 \\
\hline \multicolumn{4}{|l|}{ Apnea index (events/h) } \\
\hline Mean (SD) unadjusted & $3.8(3.6)$ & $3.1(2.4)$ & 0.61 \\
\hline Mean $(95 \% \mathrm{Cl})$ adjusted $^{\mathrm{a}}$ & $4.1(1.7-6.5)$ & $2.9(0.4-5.4)$ & 0.47 \\
\hline Mean $(95 \% \mathrm{Cl})$ adjusted $^{\mathrm{a}}$ & $38(19-57)$ & $9(-8$ to 27$)$ & 0.04 \\
\hline
\end{tabular}




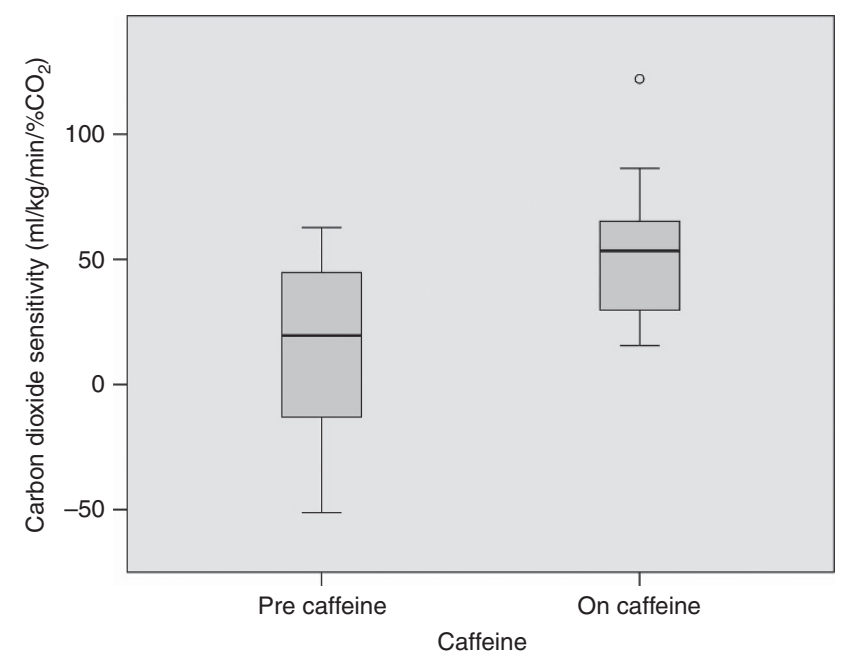

Figure 1. Box plot showing carbon dioxide sensitivity before and after administration of caffeine. The box plot shows the median and interquartile range. Whiskers extend to 1.5 times the interquartile range. Data points beyond the interquartile range are marked as outliers.

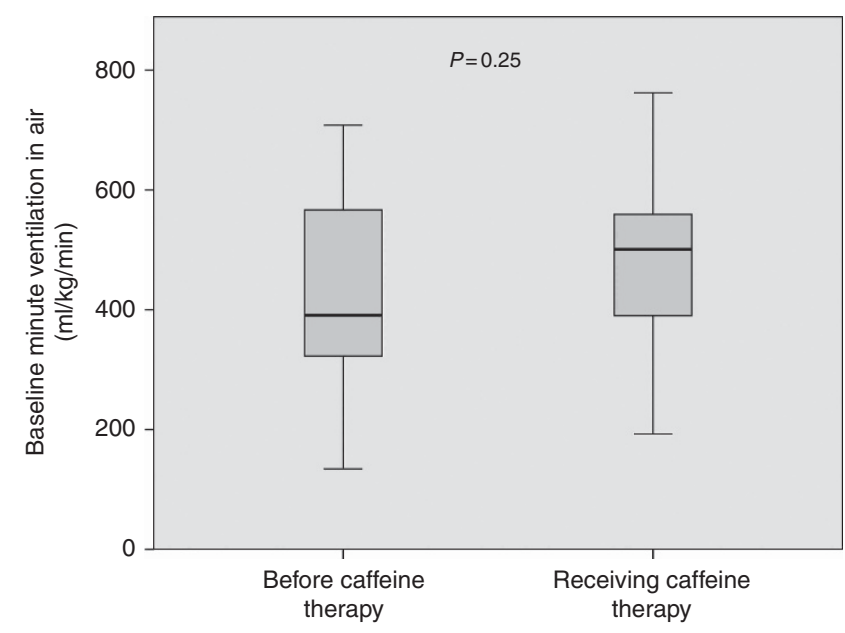

Figure 2. Box plot showing minute volume while breathing air before caffeine therapy was initiated and 1 week later. The box plot shows the median and interquartile range. Whiskers extend to 1.5 times the interquartile range. Data points beyond the interquartile range are marked as outliers.

of caffeine therapy on $\mathrm{CO}_{2}$ sensitivity at 33 weeks of gestation was $15.3 \mathrm{ml} / \mathrm{kg} / \mathrm{min} / \% \mathrm{CO}_{2}$ (95\% CI: $1-30 ; P=0.042$ ).

Infants who subsequently developed significant apnea had significantly lower birth weights $(P=0.03)$ and a trend toward lower gestational ages $(P=0.06)$ and lower $\mathrm{CO}_{2}$ sensitivities $(P=0.065)$ (Tables 1 and 2). After controlling for the effect of corrected gestational age and birth weight, $\mathrm{CO}_{2}$ sensitivity at the initial study was significantly lower in those infants who went on to develop apnea, requiring treatment with caffeine compared with those who did not (Table 2).

\section{DISCUSSION}

We have demonstrated that caffeine administration was associated with an increase in the ventilatory response to

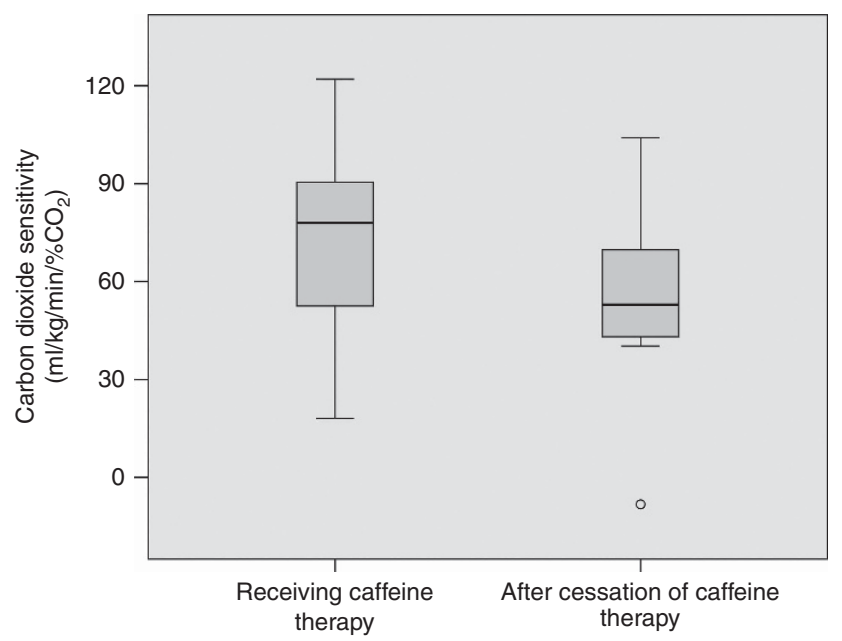

Figure 3. Boxplot showing carbon dioxide sensitivity while receiving caffeine therapy and carbon dioxide sensitivity at the following measurement 1 week later, following cessation of caffeine therapy. The box plot shows the median and interquartile range. Whiskers extend to 1.5 times the interquartile range. Data points beyond the interquartile range are marked as outliers.

hypercarbia in prematurely born infants, and that the effect remained significant after controlling for increasing maturity. When caffeine was discontinued, $\mathrm{CO}_{2}$ sensitivity significantly decreased, further suggesting that the effect was due to caffeine administration per se. Methylxanthines are phosphodiesterase inhibitors and, at therapeutic concentrations, are nonspecific adenosine receptor antagonists. Adenosine receptors are expressed throughout the brain stem respiratory centers. There are several subtypes, the blockades of which have varying effects on respiratory control (20-22). Adenosine A2A receptors play a role in the development of late hypoxic ventilatory depression, whereas A1 receptors contribute to cardiorespiratory control during normoxia (23). The role of these receptors changes with development.

The response to the initial hypercarbic challenge was significantly weaker in those who went on to develop significant apnea than in those who did not. These findings are consistent with reports that a weaker response to hypercarbia was found in infants with significant apnea (3). Furthermore, Durand et al. compared eight preterm infants with at least three episodes a day of apnea lasting for more than $20 \mathrm{~s}$ with nine preterm infants without apnea and reported reduced baseline ventilation and $\mathrm{CO}_{2}$ sensitivity in those with apnea (24). Apnea resulting in chronic intermittent hypoxia, however, may modulate the subsequent ventilator response to hypercapnea (25). Exposure of neonatal piglets to intermittent hypercapnia and hypoxia reduced the subsequent ventilatory response to hypercapnia (26). Two studies, however, in rat pups using several different regimes of hypercapnic or normocapnic hypoxia failed to demonstrate a significant effect on the subsequent ventilatory response to hypercapnia $(27,28)$. Millstrom exposed newborn rat pups to regimes of intermittent hypercapnia and hypoxia (6\% 


\section{Articles $\mid$ Rossor et al.}

$\left.\mathrm{CO}_{2} / 10 \% \mathrm{O}_{2}\right)$, or intermittent hypercapnia and hypoxia alternating with hyperoxia $\left(30 \% \mathrm{O}_{2}\right)$, five times an hour over the first 2 weeks after birth. Neither regime had any subsequent effect on the ventilatory response to hypercarbia (27). Peng used a shorter duration of intermittent hypoxia $\left(15 \mathrm{~s}\right.$ of $\left.5 \% \mathrm{O}_{2}\right)$, nine times an hour for $8 \mathrm{~h}$ a day during the first 10 days after birth in newborn rats and again showed no significant difference in their hypercapnic response compared with nonexposed controls (28). Importantly, our study has suggested that a reduced $\mathrm{CO}_{2}$ sensitivity in infants may precede the development of significant apnea and is, therefore, more likely to be a contributive factor rather than a result of recurrent apnea-induced episodes of hypercarbia and hypoxia. Whether antenatal episodes of hypercarbia may contribute is unclear. Those infants who went on to develop significant apnea were significantly more likely to be small for gestational age. Poor growth may reflect the impairment of placental blood flow. Therefore, exposure to periods of intermittent hypoxia antenatally could potentially contribute to an impairment of $\mathrm{CO}_{2}$ sensitivity.

This study has strengths and some limitations. A strength of our study is that we undertook longitudinal measurements, including infants who did not require caffeine treatment. Hence, we have been able to control for the maturational effect on the ventilatory response to hypercapnia. Our study required recruitment of infants born sufficiently prematurely that they were likely to develop apnea, but did not require respiratory support. This, then, was a highly selective group. Unfortunately, we did not recruit the target number from the sample size calculation. Nevertheless, we were able to demonstrate significant differences between groups, and given the numbers recruited, we were able to detect differences with $79 \%$ power. We did not measure caffeine levels. Absorption and metabolism of caffeine is variable in premature infants, and therefore may have introduced heterogeneity into the response to caffeine therapy. Nonetheless, the effect of caffeine on $\mathrm{CO}_{2}$ sensitivity remained significant.

In conclusion, caffeine administration was associated with an increase in the ventilatory response to hypercarbia. In addition, $\mathrm{CO}_{2}$ sensitivity assessed soon after birth was significantly lower in those infants who subsequently required treatment with caffeine for apnea.

\section{STATEMENT OF FINANCIAL SUPPORT}

The research was supported by the National Institute for Health Research (NIHR) Biomedical Research Centre based at Guy's and St Thomas' NHS Foundation Trust and King's College London.

\section{DISCLAIMER}

The views expressed are those of the authors and not necessarily those of the NHS, the NIHR, or the Department of Health.

Disclosure: J.P. is an NIHR senior investigator. All the remaining authors declare no conflict of interest.

\section{REFERENCES}

1. Abu-Shaweesh JM, Martin RJ. Neonatal apnea: what's new? Pediatr Pulmonol 2008;43:937-44.
2. Henderson-Smart DJ. The effect of gestational age on the incidence and duration of recurrent apnoea in newborn babies. Aust Paediatr J 1981;17: 273-6.

3. Gerhardt T, Bancalari E. Apnea of prematurity: I. Lung function and regulation of breathing. Pediatrics 1984;74:58-62.

4. Henderson-Smart DJ, Steer P. Methylxanthine treatment for apnea in preterm infants. Cochrane Database Syst Rev 2000;2:CD000140.

5. Mazzarelli M, Jaspar N, Zin WA, et al. Dose effect of caffeine on control of breathing and respiratory response to $\mathrm{CO}_{2}$ in cats. J Appl Physiol 1986;60:52-9.

6. Pianosi P, Grondin D, Desmond K, et al. Effect of caffeine on the ventilatory response to inhaled carbon dioxide. Respir Physiol 1994;95: 311-20.

7. D'Urzo AD, Jhirad R, Jenne $H$, et al. Effect of caffeine on ventilatory responses to hypercapnia, hypoxia, and exercise in humans. J Appl Physiol 1990;68:322-8.

8. Davi MJ, Sankaran K, Simons KJ, et al. Physiologic changes induced by theophylline in the treatment of apnea in preterm infants. J Pediatr 1978;92:91-5.

9. Fleming PJ, Levine MR, Goncalves A. Changes in respiratory pattern resulting from the use of a facemask to record respiration in newborn infants. Pediatr Res 1982;12:1031-4.

10. Rigatto H, Brady JP, de la Torre Verduzco R. Chemoreceptor reflexes in preterm infants: II. The effect of gestational and postnatal age on the ventilatory response to inhaled carbon dioxide. Pediatrics 1975;55: 614-20.

11. Ali K, Wolff K, Peacock JL, et al. Ventilatory response to hypercarbia in newborns of smoking and substance-misusing mothers. Ann Am Thorac Soc 2014;11:933-8.

12. Saiki T, Milner AD, Hannam S, et al. Sleeping position and responses to a carbon dioxide challenge in convalescent prematurely born infants studied post-termArch Dis Child Fetal Neonatal Ed 2014;99:F215-8.

13. Smith AP, Saiki T, Hannam S, et al. The effects of sleeping position on ventilatory responses to carbon dioxide in premature infants. Thorax 2010;65:824-8.

14. Prechtl HF. The behavioural states of the newborn infant (a review). Brain Res 1974;76:185-212.

15. Bhat RY, Hannam S, Pressler R, et al. Effect of prone and supine position on sleep, apneas, and arousal in preterm infants. Pediatrics 2006;118: $101-7$.

16. Corvaglia L, Zama D, Spizzichino M, et al. The frequency of apneas in very preterm infants is increased after non-acid gastro-esophageal reflux. Neurogastroenterol Motil 2011;4:303-7.

17. Berry RB, Gamaldo CE, Harding SM, et al. AASM scoring manual version 2.2 updates: new chapters for scoring infant sleep staging and home sleep apnea testing. J Clin Sleep Med 2015;11:1253-4.

18. Finer NN, Higgins R, Kattwinkel J, et al. Summary proceedings from the apnea-of-prematurity group. Pediatrics 2006;117:S47-51.

19. Bhat RY, Broughton S, Khetriwal B, et al. Dampened ventilatory response to added dead space in newborns of smoking mothers. Arch Dis Child Fetal Neonatal Ed 2005;90:F316-9.

20. Bianchi AL, Gestreau C. The brainstem respiratory network: an overview of a half century of research. Respir Physiol Neurobiol 2009;168:4-12.

21. Martin RJ, Wilson CG, Abu-Shaweesh JM, et al. Role of inhibitory neurotransmitter interactions in the pathogenesis of neonatal apnea: implications for management. Sem Perinatol 2004;28:273-8.

22. Fredholm BB. Astra Award Lecture. Adenosine, adenosine receptors and the actions of caffeine. Pharmacol Toxicol 1995;76:93-101.

23. Simakajornboon N, Kuptanon T. Maturational changes in neuromodulation of central pathways underlying hypoxic ventilatory response. Respir Physiol Neurobiol 2005;149:273-86.

24. Durand M, Cabal LA, Gonzalez F, et al. Ventilatory control and carbon dioxide response in preterm infants with idiopathic apnea. Am J Dis Child 1985;139:717-20.

25. Kinkead R, Bach KB, Johnson SM, et al. Plasticity in respiratory motor control: intermittent hypoxia and hypercapnia activate opposing 
serotonergic and noradrenergic modulatory systems. Comp Biochem Physiol A Mol Integr Physiol 2001;130:207-18.

26. Waters KA, Tinworth KD. Depression of ventilatory responses after daily, cyclic hypercapnic hypoxia in piglets. J Appl Physiol 2001;90: 1065-73.
27. Millstrom A, Kim S, Asklof K, et al. Combined effects of intermittent hyperoxia and intermittent hypercapnic hypoxia on respiratory control. FASEB J 2015;29:16.

28. Peng Y-J, Prabhakar NR. Effect of two paradigms of chronic intermittent hypoxia on carotid body sensory activity. J Appl Physiol 2004;96:1236-42. 